\title{
Electrical conductivity of modified poly(vinyl chloride)
}

\author{
MEENAKSHI MARUTHAMUTHU*, M SELVARAJ and \\ S ANNADURAI \\ Department of Physical Chemistry, University of Madras, Guindy Campus, Madras 600025, \\ India \\ MS received 7 December 1992; revised 3 May 1993
}

\begin{abstract}
Chemical modification of poly(vinyl chloride) (PVC) by dehydrochlorination with ethanolic $\mathrm{KOH}$ is found to yield modified PVC with conjugated polyene sequence. The semiconducting nature of ethoxide-modified $\mathrm{PVC}$ is illustrated with temperature dependence of conductivity $(\sigma)$. The relative ratios $(r)$ of conductivity, $\sigma_{\text {modified Pvc }} / \sigma_{\text {unmodified Pvc }}$, are greater than unity in the temperature range $50^{\circ}$ to $180^{\circ} \mathrm{C}, r$ being maximum in the vicinity of glass-transition temperature $\left(T_{\mathrm{g}}\right), T_{\mathrm{g}}$ inferred from conductivity-temperature profiles is found to be greater for modified PVC relative to unmodified PVC, which is explicable in terms of restricted free rotation limiting segmental motion. For comparison with the conductivity and $T_{\mathrm{g}}$ of ethoxide-modified PVC. LiCl-modified PVC and (aniline $+\mathrm{S}_{2} \mathrm{O}_{8}^{2-}$ )-modified PVC have also been studied.
\end{abstract}

Keywords. Electrical conductivity; poly(vinyl chloride); modified poly(vinyl chloride); glass-transition temperature; conjugated polyene sequence; rubiconjugated polymer.

\section{Introduction}

Polyconjugated systems can be obtained from saturated macromolecules by thermal, radiation and chemical methods. Thermal treatment was one of the earliest methods applied to poly(vinyl chloride) (PVC) to get conjugated polymers of improved electrical conductivity (Takarzewski 1959; Slovokhotova and Astafev 1961; Berlin et al 1962). However, thermal dehydrochlorination of PVC usually yields crosslinked polymers. Chemical modification by $\mathrm{LiCl}$ in dimethylformamide (Roth et al 1964) led to the formation of long conjugated polyenic sequences in $\mathrm{PVC}$, the resulting polymers exhibiting strong light absorption near $550 \mathrm{~nm}$ and good semiconducting behaviour with electrical conductivity of the order of $10^{-11}$ to $10^{-10} \mathrm{~S} \mathrm{~cm}^{-1}$ at room temperature. A recent patent (Ozawa et al 1986) on a highly semiconducting PVC film obtained by soaking a PVC film containing aniline in $0.1 \mathrm{~N} \mathrm{HCl}$ containing $\left(\mathrm{NH}_{4}\right)_{2} \mathrm{~S}_{2} \mathrm{O}_{8}$ also encourages studies on modification of $\mathrm{PVC}$ to get semiconducting polymers. In the present study, chemical modification of PVC has been attempted to get semiconducting polymers of the rubiconjugated type. Evaluation of the glass-transition temperature of modified PVC from conductivity data is also an objective of the study.

The significance of ethanolic $\mathrm{KOH}$ in the dehydrohalogenation of alkyl halides led us to choose this reagent for modifying PVC by dehydrochlorination. In the case of PVC, once dehydrochlorination has started, the reaction can proceed in a zipper fashion since the liberated chloride anion can catalyse the $\beta$-elimination in the neighbouring position. This would lead to the creation of blocks of conjugated polyene sequences in PVC.

\footnotetext{
* For correspondence
} 


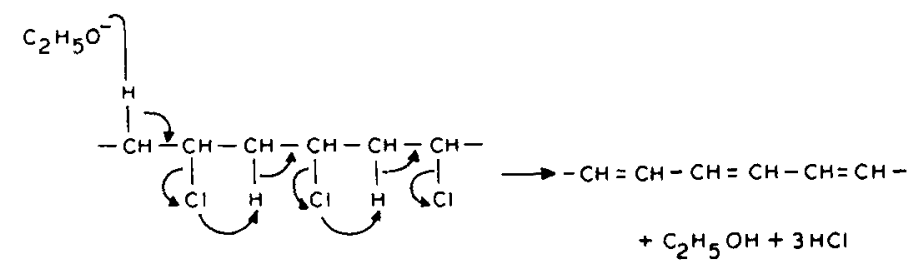

Chart 1.

If such conjugated polyene sequences are actually produced in PVC, then the resulting polymer should have a higher electrical conductivity relative to the unmodified PVC. An electrical conductivity study of PVC and ethoxide-modified PVC would therefore reveal improvement in electrical conductivity due to the introduction of such conjugated polyene sequences in PVC. It is also of interest to evaluate the glass-transition temperature $\left(T_{\mathrm{g}}\right)$ of modified PVC from conductivity-temperature profiles since a higher $T_{\mathrm{g}}$ is expected for the modified polymer than for PVC due to the restriction on the free rotation and hence restricted segmental motion. Comparison with the already known chemical modification procedures for PVC warrants preparation of modified PVC by $\mathrm{LiCl}$ method and aniline $+\mathrm{S}_{2} \mathrm{O}_{8}^{2-}$ method and conductivity study of the modified samples. Hence the present study involves chemical modification by (i) ethoxide, (ii) $\mathrm{LiCl}$ and (iii) aniline $+\mathrm{S}_{2} \mathrm{O}_{8}^{2-}$, and semiconducting behaviour of the modified polymers relative to $\mathrm{PVC}$ along with $T_{\mathrm{g}}$ evaluation from conductivity data.

\section{Experimental}

A commercial sample of PVC (SriRam Vinyls, New Delhi, India) was employed. Intrinsic viscosity $[\eta]$, of the sample, determined from dilute-solution viscosity measurements using an Ubbelhode-type viscometer corresponds to $46.3 \mathrm{ml} \mathrm{g}^{-1}$. The Mark-Houwink equation for PVC (Moore and Hutchinson 1963) in cyclohexanone at $25^{\circ} \mathrm{C},[\eta]=2.08 \times 10^{-3}[\mathrm{M}]^{0.56}$, where $[\eta]$ is given in $\mathrm{dl} \mathrm{g}^{-1}$, is used to find the molecular weight of PVC. The molecular weight of PVC thus evaluated corresponds to $9.5 \times 10^{5}$. For such a high molecular weight polymer, conductivity and $T_{\mathrm{g}}$ are independent of molecular weight. Modification of PVC by ethoxide was accomplished by stirring well a suspension of $4 \mathrm{~g}$ of $\mathrm{PVC}$ in $50 \mathrm{ml}$ of ethanolic $\mathrm{KOH}$ (a saturated solution of $\mathrm{KOH}$ in ethanol) at room temperature for about $48 \mathrm{~h}$. Filtering, washing with water, and drying in vacuum afforded the light brown polymer. For modification by $\mathrm{LiCl}, 5 \mathrm{~g}$ of $\mathrm{PVC}, 3 \mathrm{~g}$ of $\mathrm{LiCl}$ and $50 \mathrm{ml}$ of dimethylformamide (DMF) were mixed, stirred well for $24 \mathrm{~h}$ at room temperature, and poured into water, and the precipitated polymer was filtered and dried. A mixture of $5 \mathrm{~g}$ of PVC and $5 \mathrm{ml}$ of aniline in about $100 \mathrm{ml}$ of $0 \cdot 1 \mathrm{~N} \mathrm{HCl}$ was stirred for about an hour. After adding $2 \cdot 5 \mathrm{~g}$ of $\left(\mathrm{NH}_{4}\right)_{2} \mathrm{~S}_{2} \mathrm{O}_{8}$ to the above reaction mixture, stirring was continued for about $24 \mathrm{~h}$. The resulting modified polymer was filtered and dried.

For the modified PVC also, $[\eta]$ has been determined using cyclohexanone as the solvent at $25^{\circ} \mathrm{C}$. For PVC-ethoxide, $\mathrm{PVC}-\mathrm{LiCl}$ and $\mathrm{PVC}$-(aniline $\left.+\mathrm{S}_{2} \mathrm{O}_{8}^{2-}\right)$, the [ $\left.\eta\right]$ values are $37.4,75.0$ and $71.0 \mathrm{ml} \mathrm{g}^{-1}$ respectively.

Electronic spectra were recorded in the visible region using a Carl-Zeiss UV VIS spectrophotometer. IR spectra were recorded with the sample in the form of $\mathrm{KBr}$ pellets in a Perkin-Elmer 983G IR spectrometer. 
Electrical conductivity measurements were carried out using a Keithley electrometer model 614 for pressed polymer pellets in an evacuated cell (at $\sim 10^{-2}$ torr). Conductivity as a function of temperature is measured at least four times for each sample, in two heating cycles and in two cooling cycles. Generally conductivity data from the first heating cycle were higher, sometimes even two or three orders of magnitude higher, than those from the other three cycles. However, $\sigma$ from the first cooling cycle, second heating cycle and second cooling cycle have been found to be consistent and reproducible. The conductivity data from the first heating cycle have therefore been discarded. A similar observation of high $\sigma$ values for $\mathrm{LiCl}$-modified PVC in the first heating cycle has also been made earlier (Roth et al 1964).

Seebeck coefficient measurements have been made as described in the literature (Hermann and Rembaum 1967).

\section{Results and discussion}

\subsection{Electrical conductivity $(\sigma)$ and $T_{\mathrm{g}}$ of unmodified $P V C$}

PVC is an electrical insulator (Roff and Scott 1971) with $\sigma \simeq 10^{-14} \mathrm{~S} \mathrm{~cm}^{-1}$. An even lower conductivity, $10^{-17} \mathrm{~S} \mathrm{~cm}^{-1}$, has been reported elsewhere (Saito et al 1968). However, our present study on electrical conductivity of PVC (commercial sample prepared by suspension polymerization) gives a higher value of conductivity, of the order of $10^{-12} \mathrm{~S} \mathrm{~cm}^{-1}$, at room temperature. Since thermal degradation of PVC with the elimination of $\mathrm{HCl}$ commences around $200^{\circ} \mathrm{C}$, all the conductivity studies have been confined to $T<200^{\circ} \mathrm{C}$. Conductivity of unmodified PVC has been studied as a function of temperature in the temperature range $50^{\circ} \mathrm{C}$ to $180^{\circ} \mathrm{C}$. The conductivity $\sigma$ ranges from $6.74 \times 10^{-12} \mathrm{~S} \mathrm{~cm}^{-1}$ to $2.39 \times 10^{-8} \mathrm{~S} \mathrm{~cm}^{-1}$ in this temperature range (table 1). The negligible temperature dependence of conductivity in the low temperature range, $50^{\circ}$ to $80^{\circ} \mathrm{C}$, leads to an extrapolated value of conductivity at room temperature very nearly equal to $\sigma_{50 \mathrm{C}}$, i.e. $\sigma_{25 \mathrm{c}} \simeq \sigma_{50 \mathrm{c}}$. The temperature dependence of $\sigma$ and also the magnitude of $\sigma$ point to PVC being semiconducting. One of the reasons for this observed conductivity is probably in situ doping of PVC with ionic impurities during commercial preparation of PVC. Very slow thermal degradation of $\mathrm{PVC}$ at room temperature giving rise to $\mathrm{HCl}$, the ionic impurity, would also be one of the factors contributing to the observed conductivity.

Conforming to the Arrhenius-type relation

$$
\sigma=\sigma_{0} \exp \left(-E_{a} / k T\right)
$$

where $\sigma_{0}$ is a constant, $E_{a}$ the activation energy of conduction, $k$ the Boltzmann constant and $T$ the absolute temperature, the semilogarithmic plot of $\log \sigma$ vs $T^{-1}$ should be linear. However, the $\log \sigma$ vs $T^{-1}$ plot for unmodified PVC (figure 1) is not a simple linear one but is made up of three intersecting straight lines. The Arrhenius-type equation (1) is obeyed but the different activation energies in the different temperature ranges for polymer systems demands separate Arrhenius-type relations with different activation energies, $E_{a 1}, E_{a 2}$, etc. for each temperature range. The effect of temperature is not only to cause an increase in generation and transport of charge carriers but also to cause changes in the polymer itself. For example, increase in temperature may change a glassy state of an amorphous polymer to the rubbery state. A temperature 
Table 1. Electrical conductivity of PVC and modified PVC as a function of temperature.

\begin{tabular}{|c|c|c|c|c|c|}
\hline \multirow[b]{2}{*}{$T\left({ }^{\circ} \mathrm{C}\right)$} & \multirow[b]{2}{*}{ Unmodified PVC } & \multicolumn{2}{|c|}{$\sigma \times 10^{12}, \mathrm{~S} \mathrm{~cm}^{-1}$} & \multirow[b]{2}{*}{$T\left({ }^{\circ} \mathrm{C}\right)$} & \multirow[b]{2}{*}{ PVC-aniline $+\mathrm{S}_{2} \mathrm{O}_{8}^{2-}$} \\
\hline & & PVC-ethoxide & PVC-LiCl & & \\
\hline 50 & $6 \cdot 74(6 \cdot 92)$ & $25 \cdot 6$ & $9 \cdot 25$ & 35 & 16900 \\
\hline 55 & $6 \cdot 61(6 \cdot 51)$ & $27 \cdot 0$ & $12 \cdot 3$ & 40 & 20500 \\
\hline 60 & $6 \cdot 48(6 \cdot 00)$ & $29 \cdot 6$ & $15 \cdot 4$ & 45 & 22600 \\
\hline 65 & $6 \cdot 36(5 \cdot 88)$ & $32 \cdot 7$ & $16 \cdot 3$ & 50 & 27300 \\
\hline 70 & $5.96(6 \cdot 00)$ & 33.8 & $25 \cdot 4$ & 55 & 32200 \\
\hline 75 & $5 \cdot 75(5 \cdot 45)$ & $34 \cdot 6$ & $34 \cdot 4$ & 60 & 40300 \\
\hline 80 & $6 \cdot 74(6 \cdot 20)$ & 37.0 & $60 \cdot 6$ & 65 & 46100 \\
\hline 85 & $6.91(6.91)$ & 39.5 & $74 \cdot 1$ & 70 & 53700 \\
\hline 90 & $13 \cdot 1(11.95)$ & 412 & $90 \cdot 9$ & 75 & 65600 \\
\hline 95 & $19 \cdot 9(19 \cdot 9)$ & 694 & 971 & 80 & 74200 \\
\hline 100 & $43 \cdot 3(43 \cdot 0)$ & 1050 & 1650 & 85 & 86700 \\
\hline 105 & $83 \cdot 3(83 \cdot 9)$ & 1500 & 3300 & 90 & 102000 \\
\hline 110 & $136(136)$ & 2070 & 5240 & 95 & 125000 \\
\hline 115 & $297(285)$ & 3040 & 10860 & 100 & 156000 \\
\hline 120 & $581(620)$ & 4780 & 16810 & 105 & 193000 \\
\hline 125 & $1210(1220)$ & 7810 & 26530 & 110 & 225000 \\
\hline 130 & $1890(1890)$ & 10500 & 39680 & 115 & 265000 \\
\hline 135 & $2940(2940)$ & 14900 & 55560 & 120 & 297000 \\
\hline 140 & $4880(4890)$ & 20200 & 84030 & 125 & 353000 \\
\hline 145 & $6940(6950)$ & 30500 & 112360 & 130 & 412000 \\
\hline 150 & $9170(9210)$ & 44800 & 151500 & 135 & 463000 \\
\hline 155 & $12360(12300)$ & 66700 & 217400 & 140 & 549000 \\
\hline 160 & $15170(15200)$ & 90900 & 270300 & 145 & 676000 \\
\hline 165 & $17760(17800)$ & 120500 & 322600 & 150 & 826000 \\
\hline 170 & $20530(20500)$ & 128200 & 400000 & & \\
\hline 175 & $22420(22200)$ & 158700 & 476200 & & \\
\hline 180 & $23870(23400)$ & 185200 & 625000 & & \\
\hline 185 & - & 217400 & 769200 & & \\
\hline 190 & - & 243900 & 1000000 & & \\
\hline
\end{tabular}

Values of $\sigma$ in parentheses correspond to PVC with molecular weight 20,000 to 25,000

increase may also be accompanied by an order-disorder transformation or some other transition in the polymer. In other words, the effect of temperature in the case of semiconducting organic polymers is manifold relative to that in inorganic semiconductors. Since a change in conduction behaviour and activation energy of conduction would arise due to the above changes, a study of conductivity as a function of temperature would yield interesting information on these transitions in polymers. It is therefore not surprising that we get three intersecting straight lines in the semilogarithmic plot (figure 1). If we denote the two critical temperatures as $T_{c 1}$ and $T_{c 2}$ and the activation energies in the different temperature ranges as $E_{a 1}$ for $T<T_{c 1}$, $E_{a 2}$ for $T_{c 1}<T<T_{c 2}$, and $E_{a 3}$ for $T>T_{c 2}$, it is noticed that the increase in conductivity with increasing temperature in the temperature range $T<T_{c 1}$ is negligible, giving rise to the activation energy $E_{a 1} \simeq 0$. A sudden increase in conductivity is evident at $T_{c 1}$. An increase in the slope of the $\log \sigma$ vs $T^{-1}$ plot at $T>T_{c 1}$, and hence $E_{a 2}>E_{a 1}$, is evident from the figure. The sudden increase in activation energy of conduction and conductivity at a critical temperature and hence conduction behaviour, in conjunction with the fact that the glass-transition temperature of the polymer is 


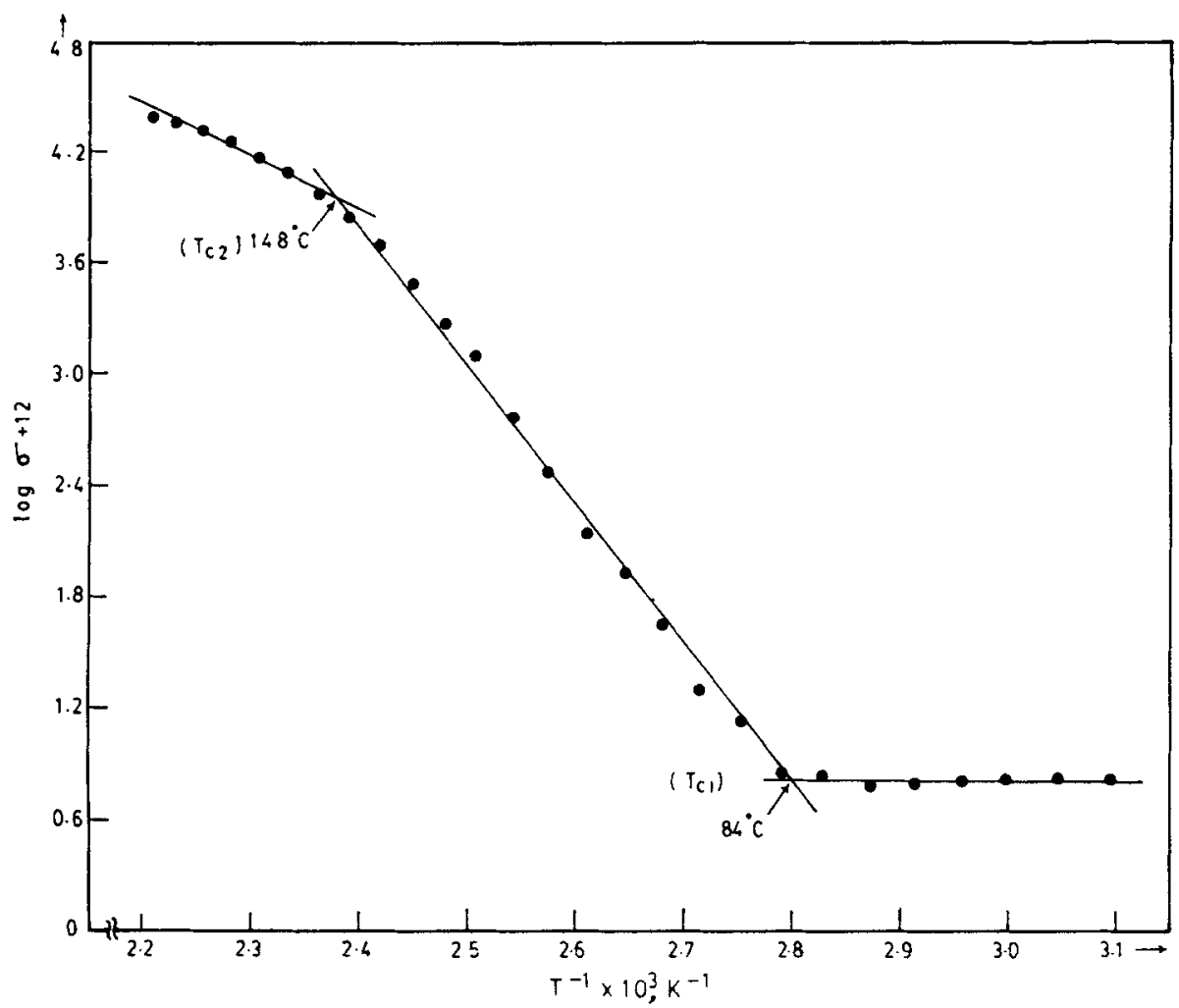

Figure 1. Conductivity-temperature profile for unmodified poly(vinyl chloride) in the form of the semilogarithmic plot. showing $T_{c 1}$ and $T_{c 2}$.

already known from a study of a basic property, makes it possible to correlate $T_{c 1}$ with $T_{\mathrm{g}}$ of the polymer as $T_{c 1}=T_{\mathrm{g}}$. The assumption made is that the activation energy of conduction in the rubbery state is greater than that ir the glassy state of the amorphous polymer. This assumption is not unreasonable considering the flexibility of the macromolecule and the mobile chain segments in the glassy state. From the point of intersection of the two linear portions with change in slope in the plot of $\log \sigma$ vs $T^{-1}, T_{c 1}$ and hence $T_{\mathrm{g}}$ is inferred to be $84^{\circ} \mathrm{C}$. This value agrees nicely with the literature value of $T_{\mathrm{g}}$ for $\mathrm{PVC}, 80^{\circ} \mathrm{C}$ to $85^{\circ} \mathrm{C}$ (Reding et al 1962; Bailey et al 1964; Barton et al 1971; Furukawa 1971; Liebman et al 1971; Usmanov et al 1971). The dielectric relaxation and conductivity studies of certain vinyl polymers including PVC (Saito et al 1968) showed that $\sigma$ for PVC ranged from $10^{-17}$ to $10^{-12} \mathrm{~S} \mathrm{~cm}^{-1}$ in the temperature range $25^{\circ} \mathrm{C}$ to $100^{\circ} \mathrm{C}$ and a break in the plot of $\log \sigma$ vs $T^{-1}$ was correlated with glass-transition temperature. $T_{\mathrm{g}}$ from their data corresponds to $70^{\circ} \mathrm{C}$, and this lower value may probably be due to the limited data in the conductivitytemperature profile.

Besides $T_{\mathrm{g}}$, another transition at $T_{c 2}$ is also observed in our study (figure 1). A second change in the slope of the $\log \sigma$ vs $T^{-1}$ plot, and hence change in activation energy, at $148^{\circ} \mathrm{C}$ indicates a transition above $T_{\mathrm{g}}$ for PVC. A transition at $T>T_{\mathrm{g}}$ at $148^{\circ} \mathrm{C}$ may be attributed to the melting of the small fraction of crystallites due to syndiotactic form (Fuller 1940; Natta and Corradini 1956), which would generally 
Table 2. Critical transition temperatures and activation energy of conduction in the various temperature ranges for PVC and modified PVC.

\begin{tabular}{lccccc}
\hline Polymer & $\begin{array}{c}T_{c 1} \\
\left.\text { (or } T_{g}\right)\left({ }^{\circ} \mathrm{C}\right)\end{array}$ & $\begin{array}{c}T_{c 2} \\
\left({ }^{\circ} \mathrm{C}\right)\end{array}$ & $\begin{array}{c}E_{a 1} \\
(\mathrm{eV})\end{array}$ & $\begin{array}{c}E_{a 2} \\
(\mathrm{eV})\end{array}$ & $\begin{array}{c}E_{a 3} \\
(\mathrm{eV})\end{array}$ \\
\hline $\begin{array}{l}\text { PVC } \\
\quad \text { (unmodified) }\end{array}$ & 84 & 148 & Negligible & 1.484 & 0.595 \\
PVC-ethoxide & 87 & 162 & 0.123 & 1.046 & 0.595 \\
PVC-LiCl & 93 & 140 & 0.577 & 1.335 & 0.773 \\
PVC-aniline $+\mathrm{S}_{2} \mathrm{O}_{\mathbf{8}}^{2-}$ & 89 & - & 0.309 & 0.454 & - \\
\hline
\end{tabular}

be present in PVC. The activation energy $E_{a 2}$ corresponds to $1.484 \mathrm{eV}\left(84^{\circ} \mathrm{C}<\right.$ $T<148^{\circ} \mathrm{C}$ ), and it is to be noted that $E_{a 3}$ is generally less than $E_{a 2}$ but greater than $E_{a 1}$ (table 2).

Another PVC sample with molecular weight distribution 20,000 to 25,000 has been studied to find out the dependence of $\sigma$ on the molecular weight of the polymer. The conductivity values are given in brackets in table 1 for this unmodified PVC. It is evident that there is negligible difference in the conductivities of the two unmodified PVC samples, showing that $\sigma$ is independent of molecular weight for a high molecular weight polymer. Furthermore, a plot of $\log \sigma$ vs $T^{-1}$ also shows the same two phase transitions at the same temperatures, showing that the phase transition temperatures are also independent of molecular weight of PVC.

\subsection{Conjugated polyene sequence in ethoxide-modified PVC}

Ethoxide-modified PVC absorbs in the visible region (figure 2) whereas unmodified PVC does not. Creation of isolated double bonds in PVC due to dehydrochiorination cannot lead to such long wavelength absorption as observed in the case of ethoxide-modified PVC. Since blocks of conjugated polyene sequences are produced in PVC, the modified polymers can, in fact, be regarded as acetylene-vinyl chloride block copolymers. The conjugated blocks produced in PVC are expected to have the absorption characteristics of simple conjugated polyenes. For example, the absorption bands for PVC modified by ethoxide can be correlated with those of a simple conjugated polyene, despite the solvent shift, as follows. The absorption spectrum of ethoxide-modified PVC (figure 2) exhibits four intense maxima, bands A to D, besides the hump at $357 \mathrm{~nm}$. The four intense bands at $370,392,418$ and $439 \mathrm{~nm}$ for the modified PVC closely parallel the ones observed for eicosadecaene (Sondheimer et al 1961), namely $370,391,413$ and $446 \mathrm{~nm}$, and the hump at $358 \mathrm{~nm}$. The close resemblance between the absorption characteristics of ethoxide-modified PVC and eicosadecaene, a simple conjugated polyene with ten double bonds, suggests that the conjugated polyene block in modified PVC is a decaene unit. The extinction coefficients $\left(\mathrm{ml} \mathrm{g}^{-1} \mathrm{~cm}^{-1}\right)$ as a function of $\lambda$ are also presented (table 3 ).

The IR spectrum of ethoxide-modified PVC shows, besides all other characteristic bands for PVC (Luther et al 1959), the $C=C$ stretching vibration of a conjugated system (Conley 1972) in the wavenumber range $1590-1640 \mathrm{~cm}^{-1}$ (figure 3), supporting the inference based on the visible absorption spectrum that conjugated polyene units are present in modified PVC. 

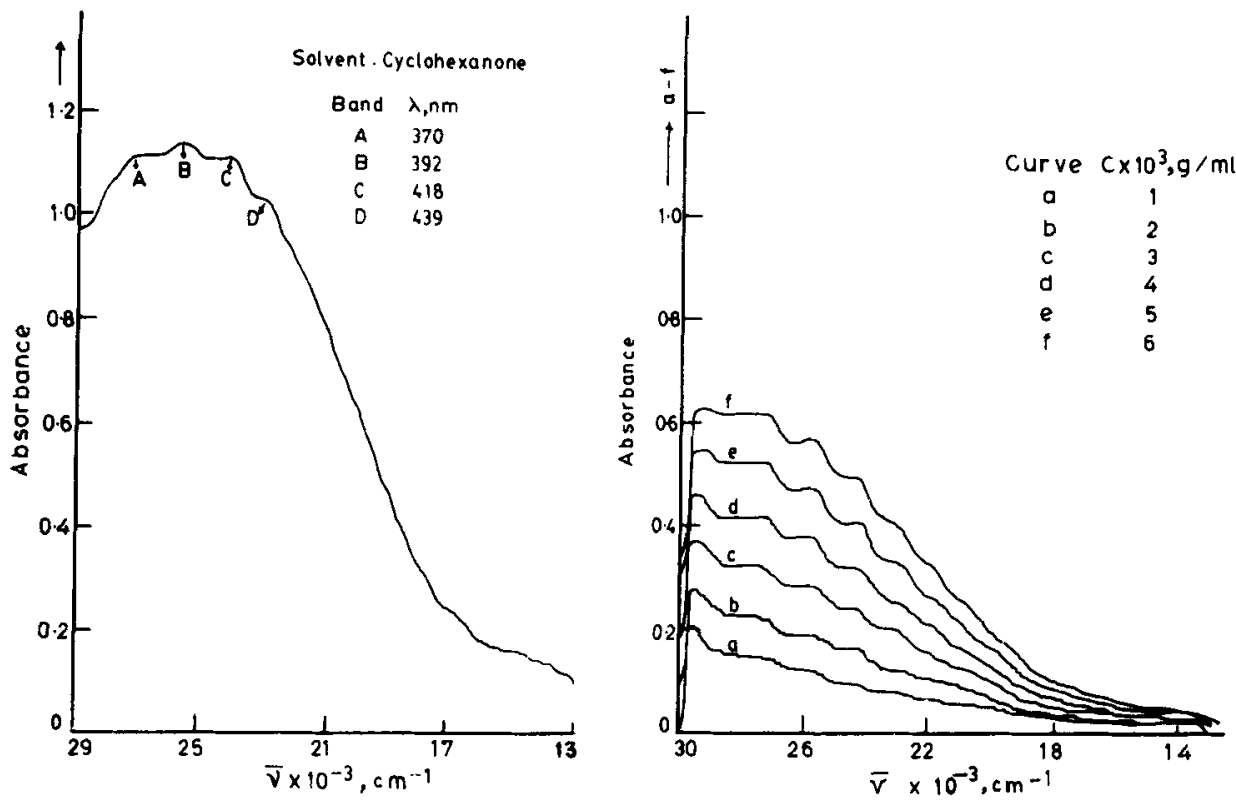

Figure 2. Visible absorption spectra of ethoxide-modified poly(vinyl chloride). Solvent: cyclohexanone. Absorbance as a function of concentration of PVC.

Table 3. Extinction coefficients as a function of $\lambda$ for modified PVC.

\begin{tabular}{|c|c|c|c|c|c|}
\hline \multicolumn{2}{|c|}{ PVC-ethoxide } & \multicolumn{2}{|c|}{ PVC-LiCl } & \multicolumn{2}{|c|}{ PVC-aniline $+\mathrm{S}_{2} \mathrm{O}_{8}^{2-}$} \\
\hline $\begin{array}{l}\lambda \\
(\mathrm{nm})\end{array}$ & $\left(\mathrm{ml} \mathrm{g}^{-1} \mathrm{~cm}^{-1}\right)$ & $\begin{array}{c}\grave{i} \\
(\mathrm{~nm})\end{array}$ & $\left(\mathrm{mlg}^{-1} \mathrm{~cm}^{-1}\right)$ & $\begin{array}{c}\lambda \\
(\mathrm{nm})\end{array}$ & $\left(\mathrm{ml} \mathrm{g}^{-1} \mathrm{~cm}^{-1}\right)$ \\
\hline 357 & 102 & 347 & 221 & 350 & 2760 \\
\hline 370 & 97 & 365 & 230 & 357 & 3100 \\
\hline 385 & 93 & 389 & 235 & 362 & 3480 \\
\hline 392 & 93 & 412 & 229 & 370 & 3510 \\
\hline 410 & 80 & 436 & 229 & 375 & 3540 \\
\hline 418 & 71 & 456 & 218 & 385 & 3140 \\
\hline 439 & 62 & 475 & 200 & 396 & 2670 \\
\hline 458 & 56 & 490 & 188 & 408 & 2110 \\
\hline 484 & 42 & 515 & 153 & 446 & 1080 \\
\hline
\end{tabular}

\subsection{Electrical conductivity and $T_{g}$ of ethoxide-modified PVC}

The electrical conductivity data as a function of temperature for ethoxide-modified PVC (table 1) show that the conductivity ranges from $2.56 \times 10^{-11}$ to $2.44 \times 10^{-7} \mathrm{~S} \mathrm{~cm}^{-1}$ in the temperature range $50^{\circ}$ to $190^{\circ} \mathrm{C}$. The conductivity increases very slowly with increasing temperature, from $50^{\circ}$ to $85^{\circ} \mathrm{C}$, the activation energy of conduction $E_{a 1}$ being $0.123 \mathrm{eV}$ in this temperature range. A sudden jump in conductivity is evident (figure 4) when the temperature increases from $85^{\circ}$ to $90^{\circ} \mathrm{C}$. On the basis of our earlier arguments, it is discernible that this critical transition temperature lying between $85^{\circ} \mathrm{C}$ and $90^{\circ} \mathrm{C}$ is the glass-transition temperature. $T_{\mathrm{g}}$ is thus found to be 

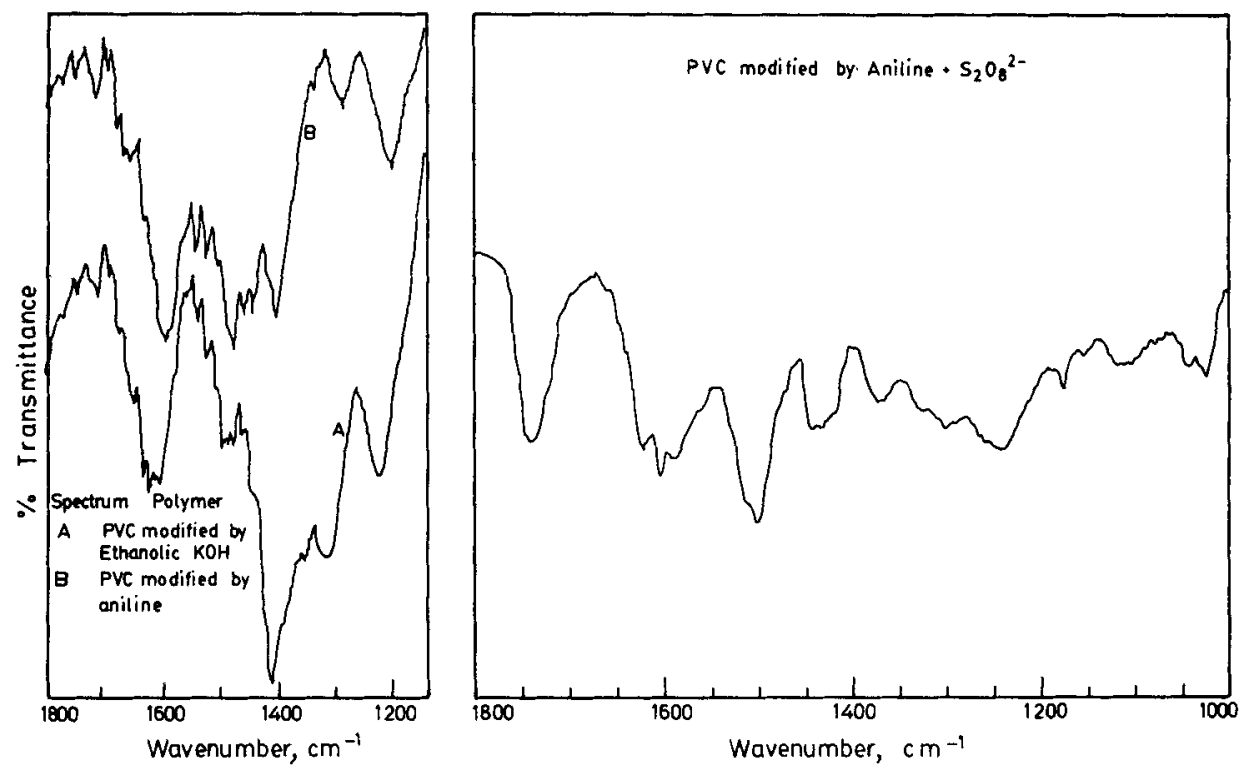

Figure 3. Infrared spectra of modified poly(vinyl chloride) in $\mathrm{KBr}$ pellet.

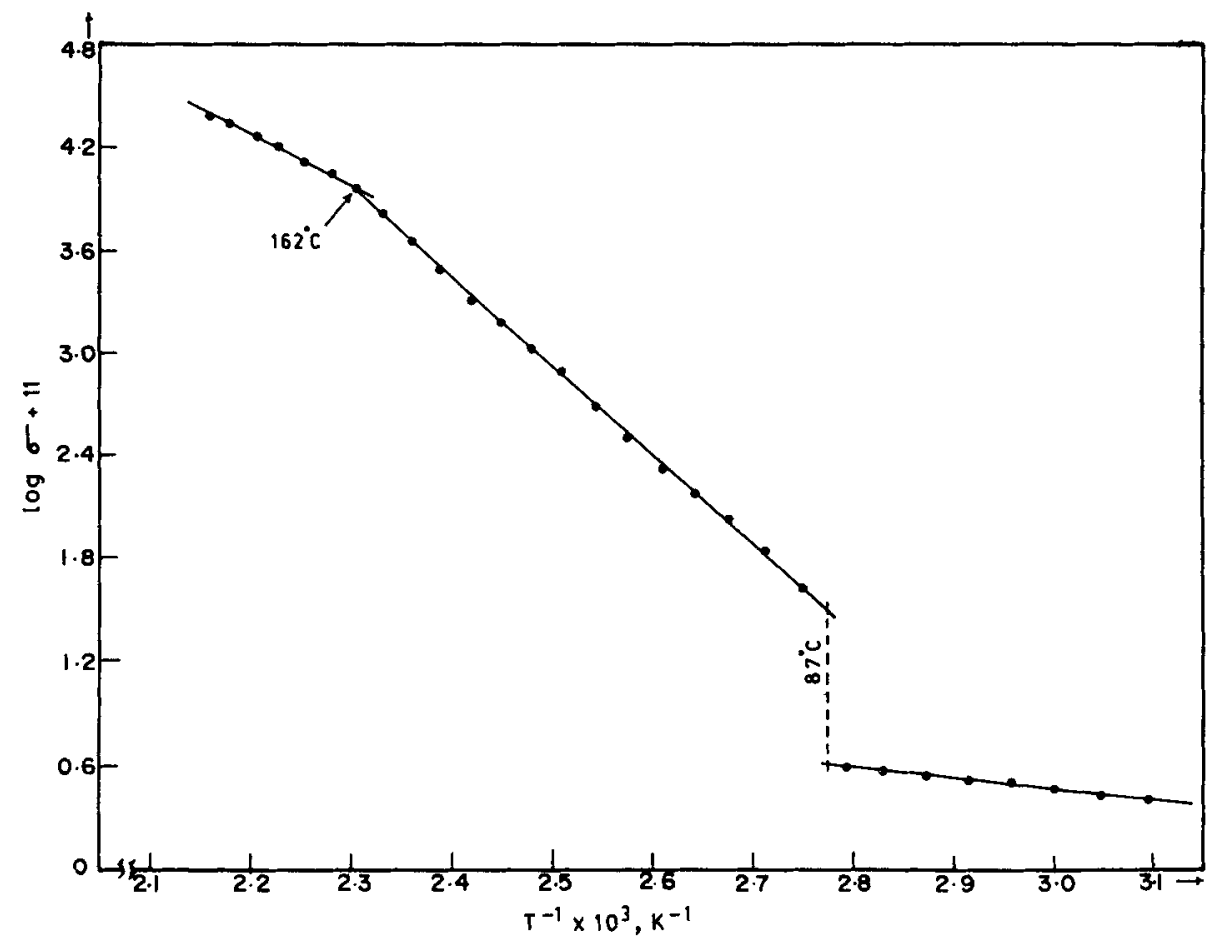

Figure 4. $\log \sigma$ as a function of $T^{-1}$ for ethoxide-modified poly (vinyl chloride). Sudden jump in conductivity at $T_{\mathrm{g}}\left(87^{\circ} \mathrm{C}\right)$ indicated by dotted line.

$87^{\circ} \mathrm{C}$ for ethoxide-modified PVC and the sudden increase in conductivity at $T_{\mathrm{g}}$, indicated by the dashed line in figure 4 , is explicable in terms of increase in free volume at $T_{\mathrm{g}}$. At $T>T_{\mathrm{g}}$, the temperature dependence of conductivity is greater (table 2), as evident from the slope of the $\log \sigma$ vs $T^{-1}$ plot. A decrease in slope at the 


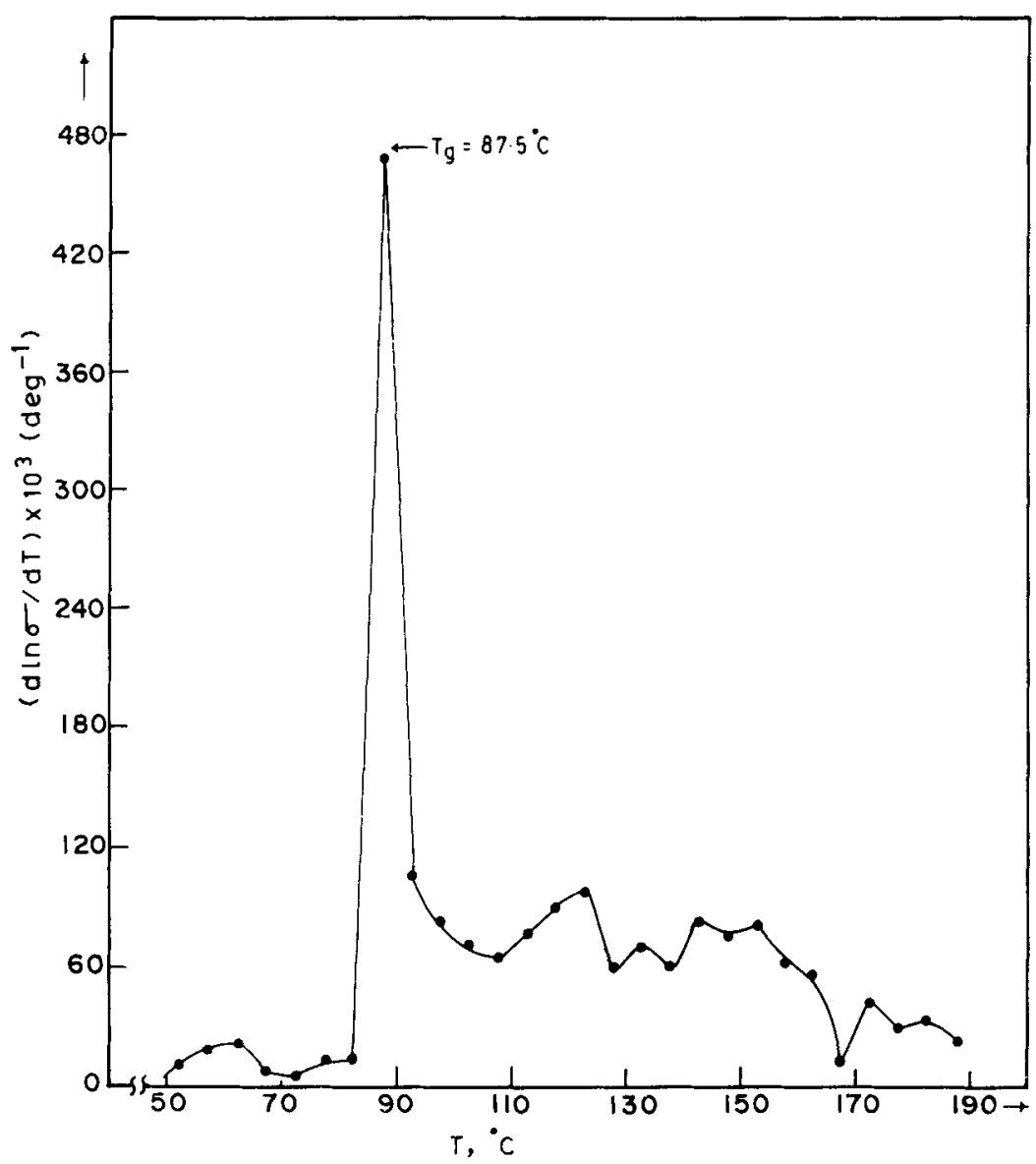

Figure 5. $\mathrm{d} \ln \sigma / \mathrm{d} T$ vs $T$ plot for ethoxide-modified poly(vinyl chloride) exhibiting maximum at $T_{\mathrm{g}}\left(87 \cdot 5^{\circ} \mathrm{C}\right)$.

second critical transition temperature of $162^{\circ} \mathrm{C}$ occurs (figure 4), giving rise to an activation energy of conduction $E_{a 3}=0.595 \mathrm{eV}$ (table 2).

Relative to unmodified PVC, ethoxide-modified PVC shows a higher conductivity and this is evident nicely from the relative ratio of $\sigma, r=\sigma_{\text {modified PVC }} / \sigma_{\text {unmodified PVC }}$. The ratio varies from 4 to 6 only in the temperature range $50^{\circ}$ to $85^{\circ} \mathrm{C}$ whereas there is a sudden increase in the vicinity of $T_{\mathrm{g}}, r$ being 32 at $90^{\circ} \mathrm{C}$ and 35 at $95^{\circ} \mathrm{C}$.

In addition to the plot of $\log \sigma$ vs $T^{-1}$, a plot of $\mathrm{d} \ln \sigma / \mathrm{d} T$ vs $T$ can also be used to evaluate $T_{\mathrm{g}}$ in the case of ethoxide-modified PVC. Since there is a sudden jump in conductivity in the vicinity of $T_{\mathrm{g}}$, the increment in conductivity, $\mathrm{d} \sigma$, for a certain increase in temperature, $\mathrm{d} T$, is found to be quite high in the glass-transition temperature range. A maximum in the plot of $\mathrm{d} \ln \sigma / \mathrm{d} T$ vs $T$ at $T=T_{\mathrm{g}}$ is therefore expected. This, in fact, is realized, as is evident from figure 5. $T_{\mathrm{g}}$ from the $\mathrm{d} \ln \sigma / \mathrm{d} T$ vs $T$ plot is found to be $87.5 \mathrm{C}$, which is in good accord with the value of $87^{\circ} \mathrm{C}$ inferred from the $\log \sigma$ vs $T^{-1}$ plot.

$T_{\mathrm{g}}$ for ethoxide-modified PVC is higher than $T_{\mathrm{g}}$ of unmodified PVC. This is explicable on the basis of the structural differences in the two polymers. Ethoxidemodified PVC, which can be regarded as a vinyl chloride-acetylene block copolymer as mentioned earlier, has conjugated polyene blocks with restricted free rotation due 
to the presence of double bonds. This would limit the segmental motion in ethoxidemodified PVC and hence the higher $T_{\mathrm{g}}$ is understandable. Since the conjugated blocks introduced are small, the increase in $T_{\mathrm{g}}$ is also small.

\subsection{Comparison with other modified PVCs}

LiCl-modified and (aniline $+\mathrm{S}_{2} \mathrm{O}_{8}^{2-}$ )-modified PVCs, which also exhibit visible absorption (figures 6 and 7) and possess conjugated polyene sequence (IR spectra in figure 3) were studied and compared with ethoxide-modified PVC. Conductivitytemperature profiles are presented for the two systems in the form of semilogarithmic plots of $\log \sigma$ vs $T^{-1}$ (figures 8 and 9). Even though the conductivity of LiCl-modified PVC is lower than that of ethoxide-modified PVC in the low temperature range $\left(50^{\circ}\right.$ to $90^{\circ} \mathrm{C}$ ), a reverse trend is noticed in the high temperature range, $\sigma_{\mathrm{PVC} \text {-LiCl }}>$ $\sigma_{\text {PVC-ethoxide }}$ (table 1). $T_{\mathrm{g}}$ of $\mathrm{LiCl}$-modified PVC is found to be $93^{\circ} \mathrm{C}$ from conductivity data, and a second critical transition temperature is also observed, $T_{c 2}=140^{\circ} \mathrm{C}$. The higher value of $T_{\mathrm{g}}$ for $\mathrm{LiCl}$-modified sample relative to ethoxide-modified sample can be interpreted in terms of the long conjugated polyene sequence in the former as evident from the $\lambda_{\max }$ value from the data of extinction coefficients as a function of $\lambda$ (table 3 ), $\lambda_{\max }$ for PVC-LiCl $>\lambda_{\max }$ for PVC-ethoxide.

PVC modified by aniline $+\mathrm{S}_{2} \mathrm{O}_{8}^{2-}$ exhibits maximum conductivity (table 1) of all the three modified PVCs. Even at $35^{\circ} \mathrm{C}$, the conductivity is found to be $1.69 \times 10^{-8} \mathrm{~S} \mathrm{~cm}^{-1}$. The room temperature value of $\sigma, \sigma_{25^{\circ} \mathrm{C}}$, corresponds to $1.1 \times 10^{-8} \mathrm{~S} \mathrm{~cm}^{-1}$. The temperature dependence of conductivity is quite low compared to other modified samples of PVC, $\sigma_{150^{\circ} \mathrm{C}}$ being only $82.6 \times 10^{-8} \mathrm{~S} \mathrm{~cm}^{-1}$ (table 1). The increase in slope at $T>T_{\mathrm{g}}$ is not as distinct as in the case of $\mathrm{PVC}-\mathrm{LiCl}$ and $\mathrm{PVC}-$-ethoxide. However it is possible to infer the $T_{\mathrm{g}}$ of the polymer to be $89^{\circ} \mathrm{C}$ (figure 9). The activation

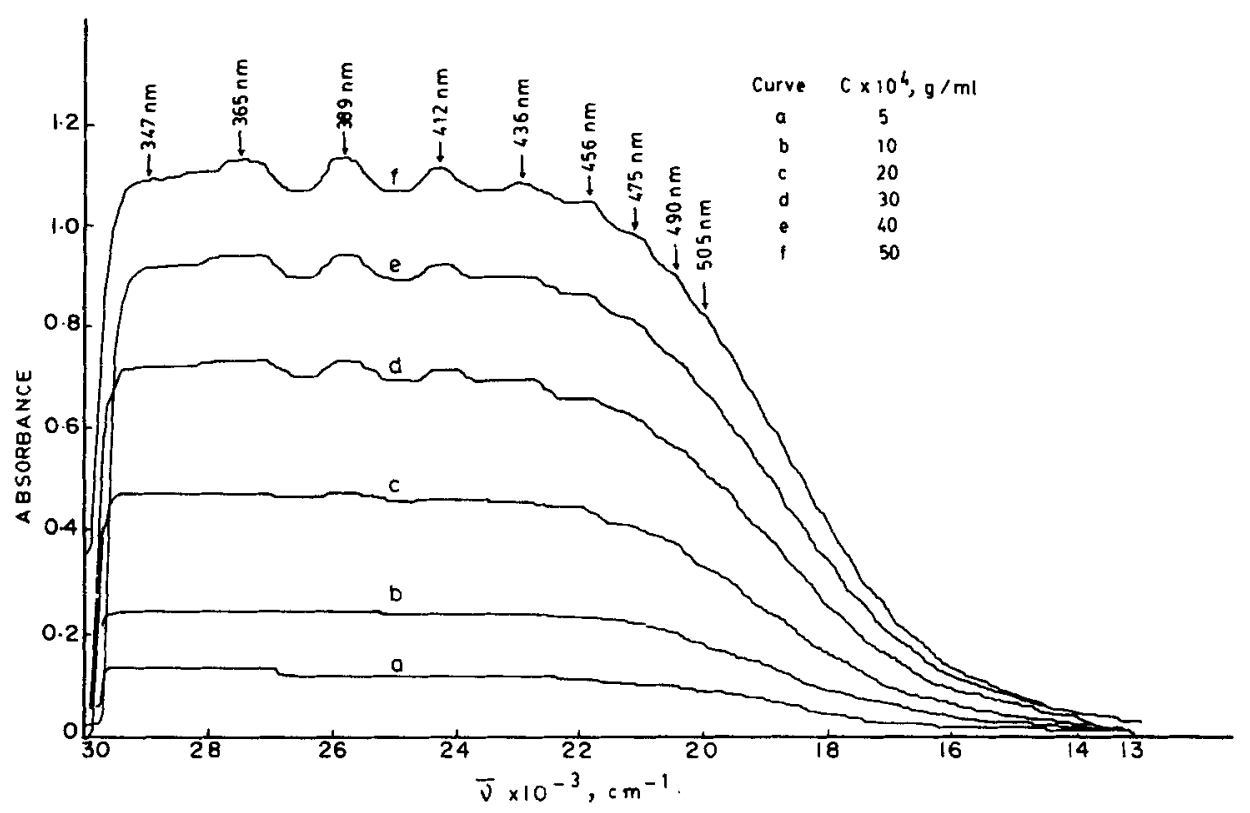

Figure 6. Visible absorption spectra of LiCl-modified poly(vinyl chloride). Solvent: cyclohexanone. Absorbance as a function of concentration of PVC. 


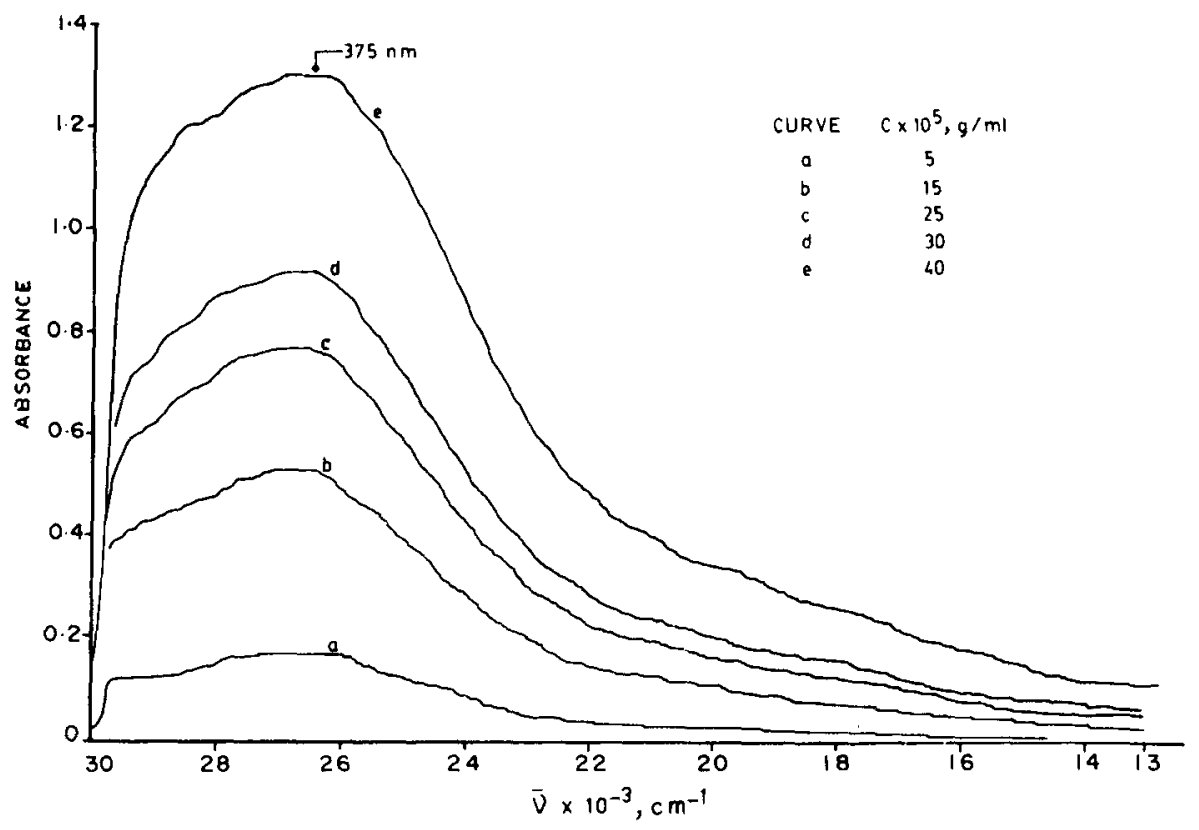

Figure 7. Visible absorption spectra of poly(vinyl chloride) modified by aniline $+\mathrm{S}_{2} \mathrm{O}_{8}^{2-}$ at different concentrations of the polymer.

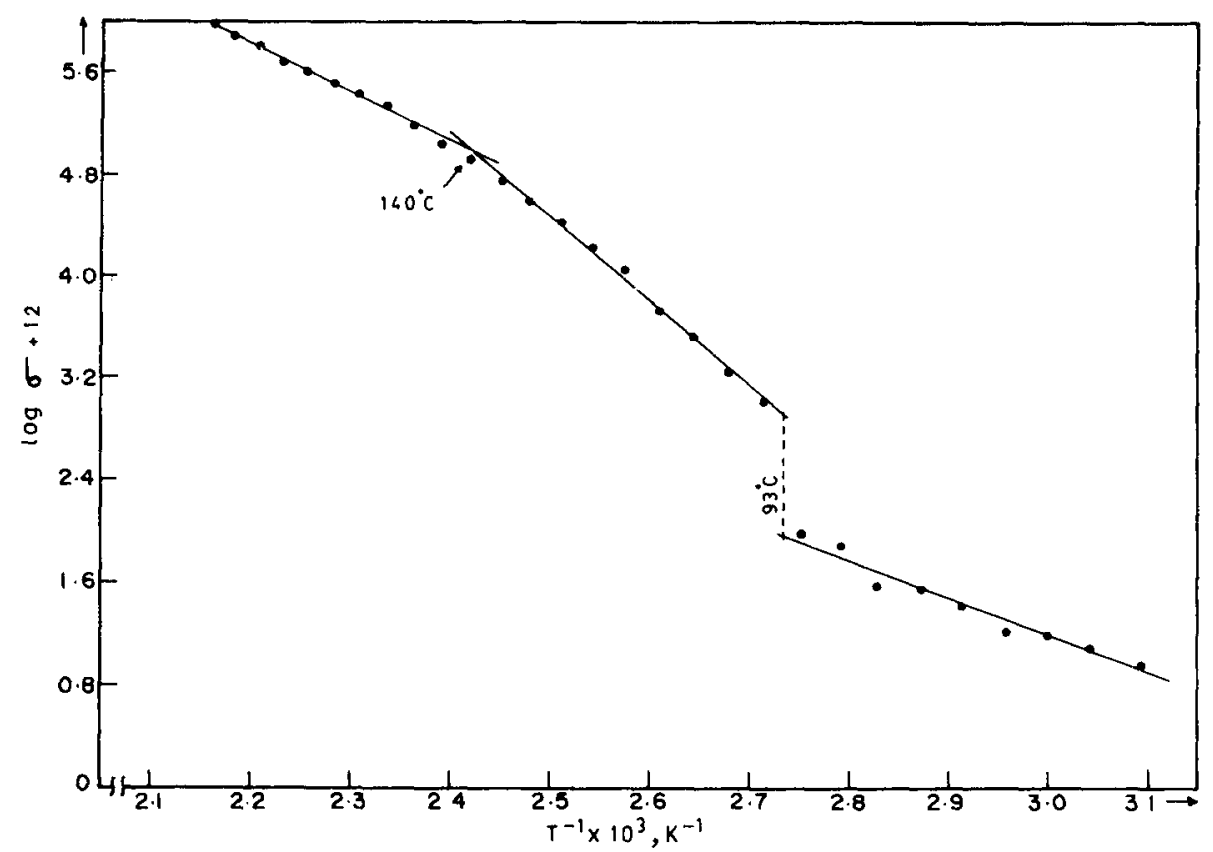

Figure 8. Conductivity-temperature profile in the form of $\log \sigma$ vs $T^{-1}$ for LiCl-modified poly (vinyl chloride). 


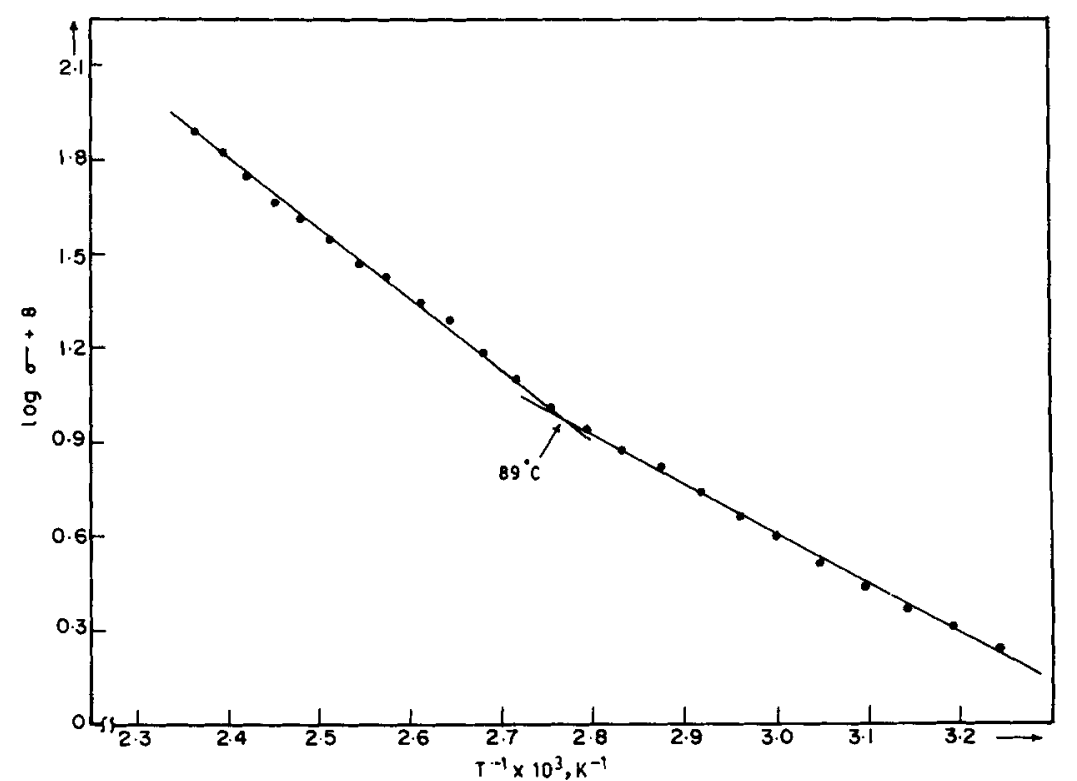

Figure 9. Conductivity-temperature profile in the form of $\log \sigma$ vs $T^{-1}$ for poly(vinyl chloride) modified by aniline $+\mathrm{S}_{2} \mathrm{O}_{8}^{2-}$.

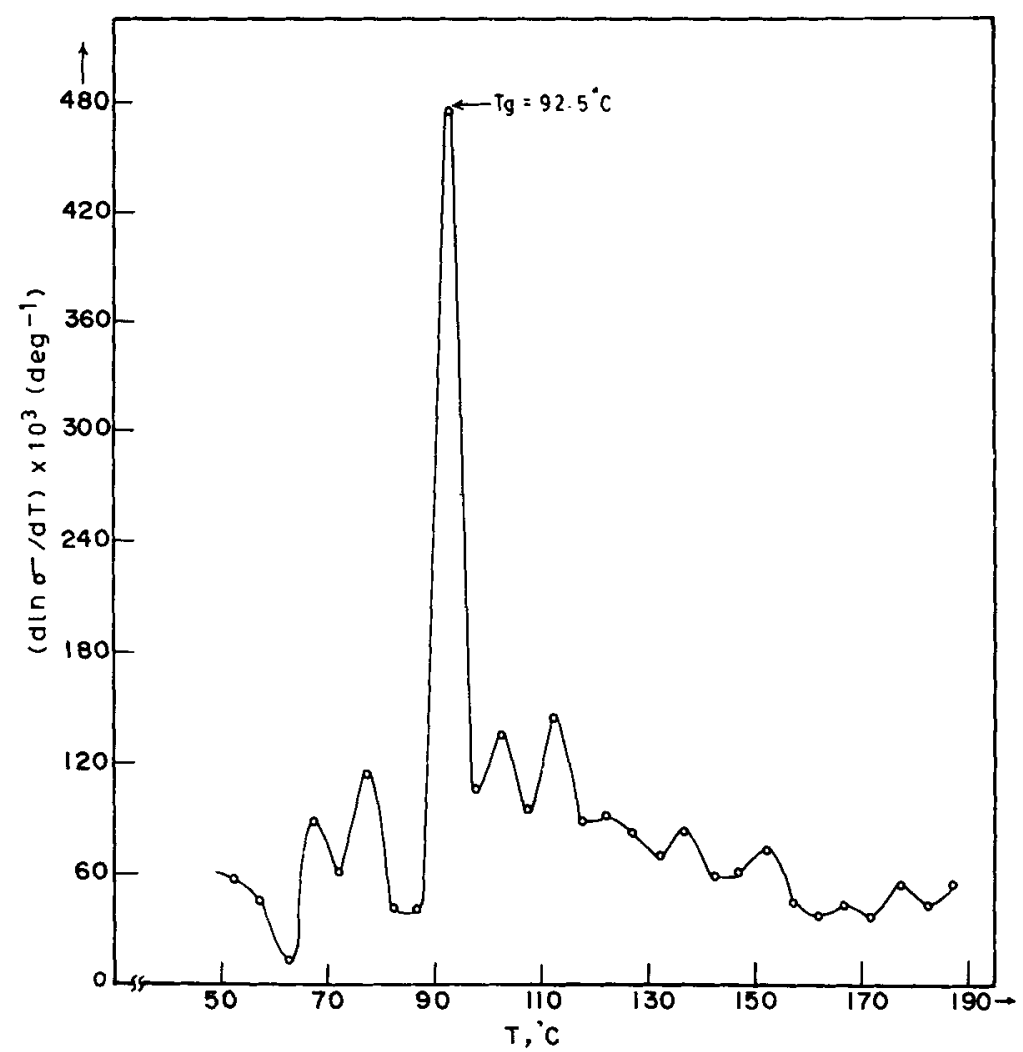

Figure 10. $\mathrm{d} \ln \sigma / \mathrm{d} T$ vs $T$ plot for LiCl-modified poly(vinyl chloride) exhibiting maximum at $T_{\mathrm{g}}\left(92 \cdot 5^{\circ}\right)$. 
energy of conduction $E_{a 2}$ at $T>T_{\mathrm{g}}$ is much less relative to $E_{a 2}$ of the other two modified samples (table 2).

The plot of $\mathrm{d} \ln \sigma / \mathrm{d} T$ vs $T$ is also utilized in the case of $\mathrm{PVC}-\mathrm{LiCl}$ to infer the $T_{\mathrm{g}}$ from the maximum in the plot (figure 10). $T_{\mathrm{g}}$ inferred thus, $92 \cdot 5^{\circ} \mathrm{C}$, shows very good agreement with $T_{\mathrm{g}}\left(93^{\circ} \mathrm{C}\right)$ from the $\log \sigma$ vs $T^{-1}$ plot.

Despite the fact that PVC film modified by aniline $+\mathrm{S}_{2} \mathrm{O}_{8}^{2-}$ gave rise to a highly semiconducting film (Ozawa et al 1986) with $\sigma$ of the order of $10^{-2} \mathrm{~S} \mathrm{~cm}^{-1}$, the same reagents used for powdered PVC yielded modified PVC with $\sigma$ only of the order of $10^{-8} \mathrm{~S} \mathrm{~cm}^{-1}$ in the present work. The difference in conductivity would be due to different extents of conjugation in the modified sample, which in turn stems from the difference in the concentrations of the reagents and other experimental conditions used in the preparation of modified PVC.

Relative to LiCl-modified PVC prepared earlier (Roth et al 1964), LiCl-modified PVC prepared by us exhibits a lower conductivity, by nearly an order of magnitude. This is attributed once again to the longer polyenic sequences in the former compared to the latter. Roth et al (1964) observed only one critical transition temperature which ranges from $80^{\circ} \mathrm{C}$ to $104^{\circ} \mathrm{C}$ for the modified PVC with different percentages of conjugated double bonds. This is probably due to limiting the conductivity study up to $\sim 150^{\circ} \mathrm{C}$. The relative magnitudes of $E_{a 2}$ and $E_{a 1}, E_{a 2}>E_{a 1}$, observed by them also confirm the validity of the assumption made in the evaluation of $T_{\mathrm{g}}$ in the present work, $E_{a}$ in the rubbery state of the polymer is $>E_{a}$ in the glassy state of the polymer. A higher activation energy for the rubbery state of the polymer relative to the glassy state has also been observed in the case of elastomeric ionenes (Somoano et al 1970).

Ethoxide-modified PVC as well as the other two samples of modified PVC prepared and studied for comparison with the former belong to the class of rubiconjugated polymers with limited conjugation. Hence the improvement in conductivity is also subject to limitation. The advantageous properties of PVC coupled with this semiconducting nature may however prove to be fruitful.

\subsection{Seebeck coefficients of modified PVC}

Measurements of Seebeck coefficients for the modified PVCs show that they exhibit very large and negative Seebeck coefficients $(Q)$ of the order of $\mathrm{mV} \mathrm{K}^{-1}$. For example,

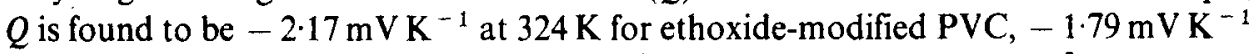
at $316 \mathrm{~K}$ for $\mathrm{PVC}-\mathrm{LiCl}$ and $-1.96 \mathrm{mV} \mathrm{K}^{-1}$ at $319 \mathrm{~K}$ for (aniline $+\mathrm{S}_{2} \mathrm{O}_{8}^{2-}$ )-modified PVC, which are closely related. The data suggest that electrons are the majority carriers for the transport process and the modified PVCs are $n$-type semiconductors. PVC modified by $\mathrm{LiCl}$ shows a transition from $n$-type to $p$-type semiconductivity at high temperatures, $\sim 425 \mathrm{~K}$.

\section{References}

Bailey F E, Henry J P, Lundberg R D and Whelan J M 1964 J. Polym. Sci. B2 447

Barton J, Pegoraro M, Szilagyi L and Pagani G 1971 Makromol. Chem. 144245

Berlin R M, Aseeva R M, Kamchev G I and Frankevich E L 1962 Dokl. Akad. Nauk SSSR 1441042

Conley R T 1972 in Infrared spectroscopy (Boston: Allyn and Bacon Inc) p. 109

Fuller C S 1940 Chem. Rev. 26243

Furukawa J 1971 Pure Appl. Chem. 26153 
Hermann A M and Rembaum A 1967 J. Polym. Sci. C17 107

Liebman S A, Foltz C R, Reuwer J F and Obremski R J 1971 Macromolecules 4134

Luther H, Meyer H and Loew H 1959 Z. Anal. Chem. 170155

Moore W R and Hutchinson R J 1963 Nature (London) 2001095

Natta G and Corradini P 1956 J. Polym. Sci. 20251

Ozawa S, Hagiwara T and Iwata K 1986 Jpn. Patent 61/123 638; 1987 Chem. Abstr. 1066150 c

Reding F R, Water E R and Welch F J 1962 J. Polym. Sci. 56225

Roff W J and Scott J R 1971 in Fibres, films and plastics-A handbook of common polymers (London: Butterworths) p. 575

Roth J P, Rempp P and Parrod J 1964 J. Polym. Sci. C4 1347

Saito S, Sasabe H, Nakajima T and Yada K 1968 J. Polym. Sci. Polym. Lett. Edn. 61297

Slovokhotova N A and Astarev I V 1961 Vyskomol. Soedin 31607

Somoano R, Yen S P S and Rembaum A 1970 J. Polym. Sci. Polym. Lett. Edn. 8467

Sondheimer F, Ben-Efraim D A and Wolovsky R $1961 \mathrm{~J}$. Am. Chem. Soi. 831675

Takarzewski L 1959 Roczniki. Chemii. 33619

Usmanov K V, Yulchibaev A A, Asamov M K and Valiev A 1971 J. Polym. Sci. A19 1459 